\title{
Pareto optimization using the method of ant colony
}

\author{
Olga Chengar ${ }^{1, *}$, Elena Savkova $^{2}$,Elena Vladimirova ${ }^{1}$, and Nikolay Sapozhnikov ${ }^{3}$ \\ ${ }^{1}$ Sevastopol State University, Department of Information Technologies and Computer Systems, 299053, University Str., Sevastopol, \\ Russian Federation \\ ${ }^{2}$ Donetsk National Technical University, Department of Automated Control Systems, 83084, Artyoma Str., Donetsk, Ukraine \\ ${ }^{3}$ Federal state owned military educational institution of the Higher professional Education "the black sea higher navy order of the red \\ star academy by P.S.Nakhimov", 299028, Dybenko Str., Sevastopol, Russian Federation
}

\begin{abstract}
The article deals with the development of an effective mathematical and algorithmic apparatus for solving multicriteria problems of optimizing scheduling in engineering using the Pareto principle. The proposed algorithm uses the instrumental capabilities of the ant colony method, which makes it possible to obtain the solution closest to the "preferred" point of the criteria space that is given by the user. The variant of forming the objective function as a weighted sum of separate criteria is considered.
\end{abstract}

\section{Introduction}

The real optimization problem is usually solved for several criteria. A number of scientific works and publications have been devoted to this issue [1-5]. But the problem of constructing the Pareto set approximation (and thus of the Pareto front) is not completely solved. This problem lies in the fact that the standard methods of multicriteria optimization do not allow determining the whole set of effective solutions, especially in the scheduling tasks for machine-building production. To use even the simplest of such methods with high accuracy Pareto set approximation and / or high computational complexity of target functions, generally unacceptably high computational resources are required. Therefore, at present, alternative methods are developing intensively.

Usually, these algorithms are based on evolutionary algorithms and, more often, on genetic algorithms [6]. Then the corresponding Pareto-approximation methods are called evolutionary, and in the area under consideration they are the most promising developments. The basic principle of these methods is not the use of evolutionary algorithms, but the rules for the formation of fitness functions that ensure the movement of individuals in the population, ultimately, in the direction of the Pareto set $[6,7]$. The evolution of these individuals can proceed according to laws different from the laws used in evolutionary algorithms, for example, according to the laws motion of the ant population in the ant algorithm.

Thus, the task of multicriteria optimization, based on the so-called "population" Pareto-approximation methods, becomes urgent. The aim of the work is the development of an effective mathematical and algorithmic apparatus for solving multicriteria optimization problems on the basis of the ant colony method, which can easily be adapted to given conditions with additional constraints in the problem.

\section{The research task formulation}

Multicriteria optimization is based on finding a solution that is the best at the same time for several functions $[8,9]$. To this end, special methods have been developed for to differ significantly from the standard technique, which has been focused on the optimization of one function. While using the Pareto optimization approach for multicriteria problems, there are two types of search in target space [10]:

1) constant search direction (in the case of fixed weights in the target function);

2) multiple search direction.

In general, the problem of multicriteria optimization can be formulated as follows (1):

$$
\begin{gathered}
\max (\min )\left\{\mathrm{z}_{1}=\mathrm{f}_{1}(\mathrm{x}), \mathrm{z}_{2}=\mathrm{f}_{2}(\mathrm{x}), \ldots, \mathrm{z}_{\mathrm{q}}=\mathrm{f}_{\mathrm{q}}(\mathrm{x})\right\} \\
\mathrm{g}_{\mathrm{i}}(\mathrm{x}) \leq 0 ; \quad \mathrm{x} \geq 0 ; \quad \mathrm{i}=1,2, \ldots, \mathrm{m}
\end{gathered}
$$

where $\mathrm{f}_{\mathrm{q}}(\mathrm{x})$ is a function-criterion; $\mathrm{q}$ is number of criteria; $\mathrm{g}_{\mathrm{i}}(\mathrm{x})$ is task limitation; $\mathrm{m}$ is the number of constraints.

Here, the solution search space is defined (2):

$$
S=\left\{x \in R^{n} \mid g_{i}(x) \leq 0, \quad i=1,2, \ldots, m, x \geq 0\right\}
$$

where $\mathrm{x} \in \mathrm{R}^{\mathrm{n}}$ is vector of variable parameters.

In the case of multicriteria optimization, graphical interpretation in the space of criteria is often used Z (3).

$$
Z=\left\{z \in R^{q} \mid z_{1}=f_{1}(x), z_{2}=f_{2}(x), \ldots, z_{q}=f_{q}(x), x \in S\right\}
$$

where $\mathrm{z} \in \mathrm{R}^{\mathrm{q}}$ is values vector of $\mathrm{q}$ target functions.

Thus, $\mathrm{Z}$ is the set of images in $\mathrm{S}$ (fig. 1 and fig. 2).

\footnotetext{
* Corresponding author: OVChengar@sevsu.ru
} 


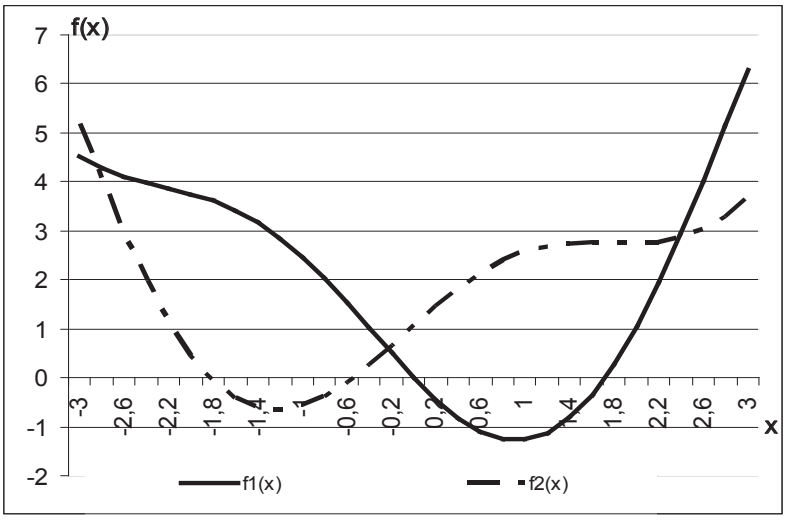

Fig.1. Target functions in the solution space $\mathrm{S}$.

If the solution is not dominated by any other solution, then it is called non-dominant or optimal in the sense of Pareto. It should be noted that multicriteria tasks are fundamentally different from single-criteria ones [10]. In the latter case, it is necessary to find a solution that is better than all the others. In the case of multicriteria optimization, there is not necessarily a solution that is the best with respect to all the criteria separately due to possible conflicts.

The decision can be better with respect to one criterion and worse with respect to other criteria [7, 10]. According to Pareto's dominance principle, the potential solutions of the multicriteria problem are divided into dominated and non-dominated solutions [10].

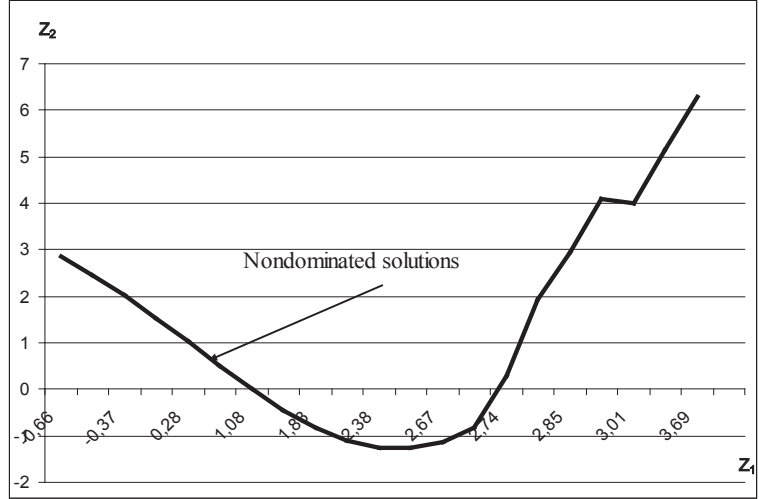

Fig.2. Target functions in the space of criteria Z.

A solution $\mathrm{X}$ is said to be dominant if there exists a solution $\mathrm{Y}$ that is not worse than $\mathrm{X}$ by all criteria, that is, for all optimizable functions $f_{i}(i=1, \ldots, q): f_{i}(x) \leq f_{i}(y)$ for all $1 \leq \mathrm{i} \leq \mathrm{k}$ while maximizing the function $\mathrm{f}_{\mathrm{i}}$ and $f_{i}(x) \geq f_{i}(y)$ for all $1 \leq \mathrm{i} \leq \mathrm{k}$ while minimizing the function $f_{i}$. Algorithms, which are based on ant colonies do not impose any requirements on the kind of target functions and constraints and allow to obtain a lot of potential solutions in various directions of global search. Therefore, in case of multicriteria optimization, the search for more than one path of ants' population movement along the graph vertexs is performed, and multiple paths for each ants population optimal in the sense of Pareto are searched. Then the general structure of the multicriteria approach based on the method ant's colonies can be represented by the following algorithm (fig. 3).

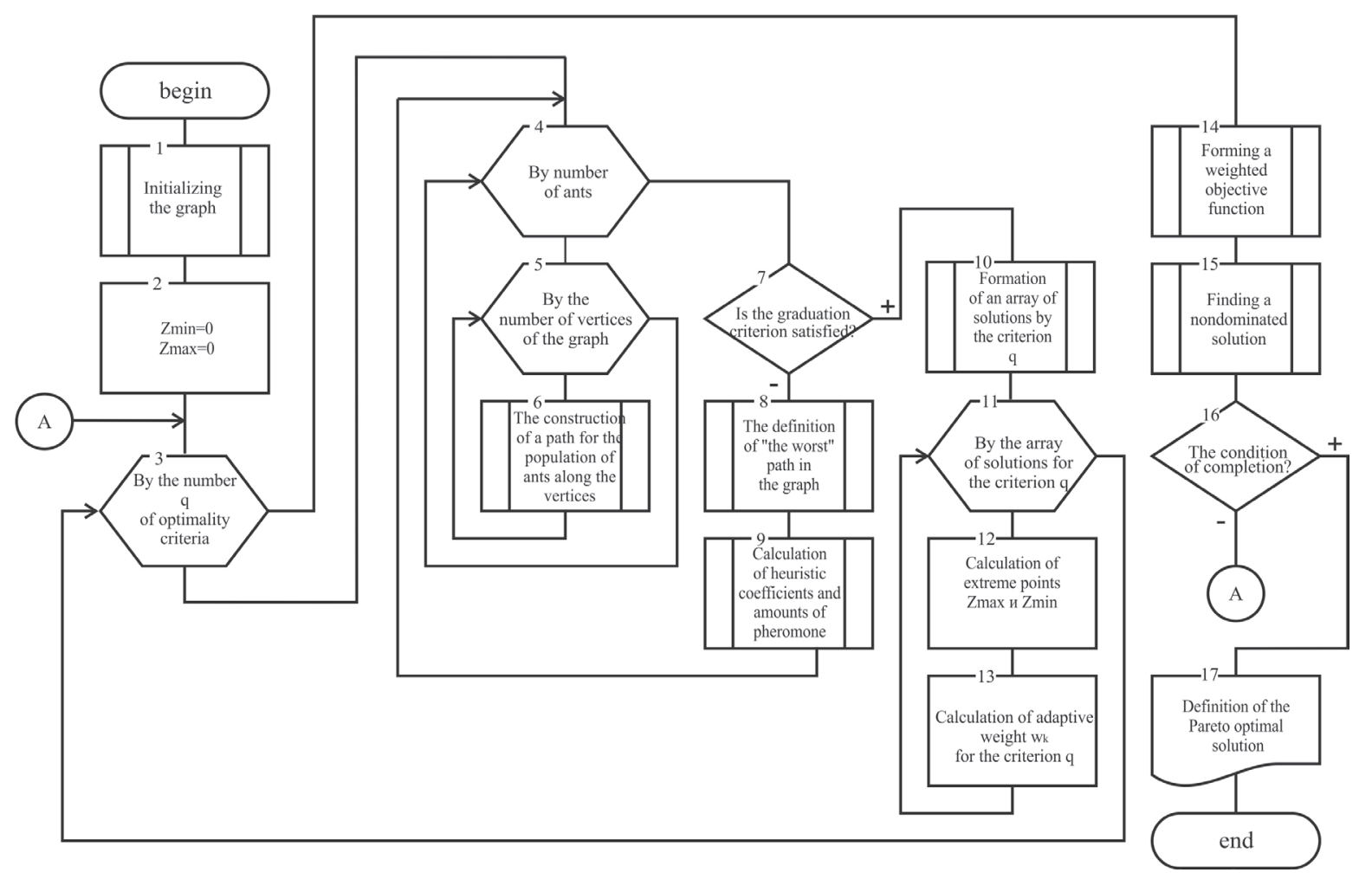

Fig.3. Generalized algorithm for solving a multicriteria problem based on the method ant's colonies. 
In fact, the method ant's colonies refers to the methods of meta-strategy, therefore, in order to solve a specific problem, in addition to the basic parameters the ant algorithm, it is necessary to select and justify the basic criteria for optimality.

Justification of this choice or that criterion of efficiency is responsible and far from always obvious task. The difficulty is in that the various optimality criteria are often contradictory, optimization by one criterion leads to a deterioration in quality by another criterion. There are several ways to solve this problem and the main one is the reduction of the multicriteria problem to the one-criterion one, for example, using the integrated criterion, as the sum of particular criteria with some expertly assigned coefficients (the so-called additive convolution method). However, such an approach also requires a serious justification, first of all, because of the subjective arbitrariness in choosing the structure of the integrated criterion and the appointment of coefficients.

Since the multicriteria optimization is a natural development of ordinary numerical or combinatorial optimization, many developed methods have been extended to this more general case [7]. While using the ant algorithm for multicriteria optimization, the central question is the construction of the objective function $[11,12]$. Recently, with the rapid development of evolutionary and population optimization methods, several approaches have been developed $[13,14]$, one of which is the weighted sum method, the essence of which is to determine the weight of each criterion included in the objective function [15].

This approach is one of the most popular and natural development of classical optimization methods, in which the "general" target function is constructed from separate target functions in the form of a weighted sum (4).

$$
F(x)=\sum_{i=1}^{k} w_{i} f_{i}(x)
$$

where weights $\mathrm{w}_{\mathrm{i}} \in[0,1]$ and $\sum_{\mathrm{i}=1}^{\mathrm{k}} \mathrm{w}_{\mathrm{i}}=1$

Here, each target function $\mathrm{f}_{\mathrm{i}}(\mathrm{x})$ is assigned its own weight $\mathrm{w}_{\mathrm{i}}$ and the problem reduces to the scalar case. Different values the weights $\mathrm{w}_{\mathrm{i}}$ lead to different solutions in the sense of Pareto. The scalar value of the new target function is then calculated by summing the weighted values $q$ of the optimality criteria. For parallel search for multiple solutions, weights are not fixed, which makes it possible for the ant algorithm to expand the front in all directions.

In the weighted sum method, weight determination is performed either randomly, which makes it difficult to find a Pareto optimal solution, or with the use of an adaptive weighted approach.

In the presented algorithm, at each iteration for a certain optimization criterion, a lot of decisions are formed based on the functioning the ant's algorithm. Further, for the solutions being investigated, the maximum and minimum extremal points in the space of given criteria are determined (5):

$$
\begin{aligned}
& \mathrm{z}^{+}=\left\{\mathrm{z}_{1}^{\max }, \mathrm{z}_{2}^{\max }, \ldots, \mathrm{z}_{\mathrm{q}}^{\max }\right\} \\
& \mathrm{z}^{-}=\left\{\mathrm{z}_{1}^{\min }, \mathrm{z}_{2}^{\min }, \ldots, \mathrm{z}_{\mathrm{q}}^{\min }\right\}
\end{aligned}
$$

For each criterion, the maximum and minimum values are determined as follows (6):

$$
\begin{aligned}
& \mathrm{z}_{\mathrm{k}}^{\max }=\max \left\{\mathrm{f}_{\mathrm{k}}(\mathrm{x}) \mid \mathrm{x} \in \mathrm{P}\right\}, \mathrm{k}=1,2, \ldots, \mathrm{q} \\
& \mathrm{z}_{\mathrm{k}}^{\min }=\min \left\{\mathrm{f}_{\mathrm{k}}(\mathrm{x}) \mid \mathrm{x} \in \mathrm{P}\right\}, \mathrm{k}=1,2, \ldots, \mathrm{q}
\end{aligned}
$$

where $\mathrm{z}_{\mathrm{k}}^{\min }$ and $\mathrm{z}_{\mathrm{k}}^{\max }$ are minimum and maximum value for the $\mathrm{k}$-th target according to the specified criterion; $\mathrm{q}$ is number of optimality criteria; $\mathrm{P}$ is set of solutions by a given criterion.

As a result, we obtain a hyperplane defined by two extremal points, and which contains all the current solutions. These two extremal points are updated at each iteration. The adaptive weight of the k-th goal is determined by the relation (7).

$$
\mathrm{w}_{\mathrm{k}}=\frac{1}{\mathrm{z}_{\mathrm{k}}^{\max }-\mathrm{z}_{\mathrm{k}}^{\min }}, \mathrm{k}=1,2, \ldots, \mathrm{q}
$$

For each goal, based on the selected performance criteria, average weighted weighting factors of significance are established, which are normalized within the group (8).

$$
\mathrm{w}_{\mathrm{k}}^{\text {норм. }}=\frac{\mathrm{w}_{\mathrm{k}}}{\sum_{\mathrm{i}=1}^{\mathrm{q}} \mathrm{w}_{\mathrm{i}}}, \mathrm{k}=1,2, \ldots, \mathrm{q}
$$

Then, at each iteration, the weighted target function is determined according to the following expression (9).

$$
\mathrm{z}(\mathrm{x})=\sum_{\mathrm{k}=1}^{\mathrm{q}} \mathrm{w}_{\mathrm{k}}^{\text {норм. }}\left(\mathrm{f}_{\mathrm{k}}(\mathrm{x})-\mathrm{z}_{\mathrm{k}}^{\min }\right)
$$

The schematically proposed algorithm can be represented in the form a graph (fig. 4) reflecting the sequence of constructing an adaptive moving line in the space of optimality criteria $\mathrm{Z}$ in order to search for Pareto-optimal solutions.

Initially, the algorithm assumes zeroing of all values $Z^{\min }$ and $Z^{\max }$. Since extreme points are updated at each iteration, the weights are updated accordingly. Expression (9) represents a hyperplane defined by the following extremal points in the set of current solutions (10).

As shown in fig. 4, an adaptive moving line is determined by extremal points $\left(\mathrm{z}_{1}^{\max }, \mathrm{z}_{2}^{\mathrm{min}}\right)$ and $\left(\mathrm{z}_{1}^{\min }, \mathrm{z}_{2}^{\max }\right)$. Thus, a rectangle defined by extremal points $\left(z_{1}^{\max }, z_{2}^{\min }\right)$ and $\left(\mathrm{z}_{1}^{\min }, \mathrm{z}_{2}^{\max }\right)$, is the minimum rectangle that contains all the current solutions. All 
explored Pareto solutions lie in the space $\mathrm{Z}$ and during the process of finding a solution for each population of ants, the hyperplane successively approaches a positive (or negative) ideal point. So this method allows you to adjust the weights of the objective function and directs the search for solutions in the right direction.

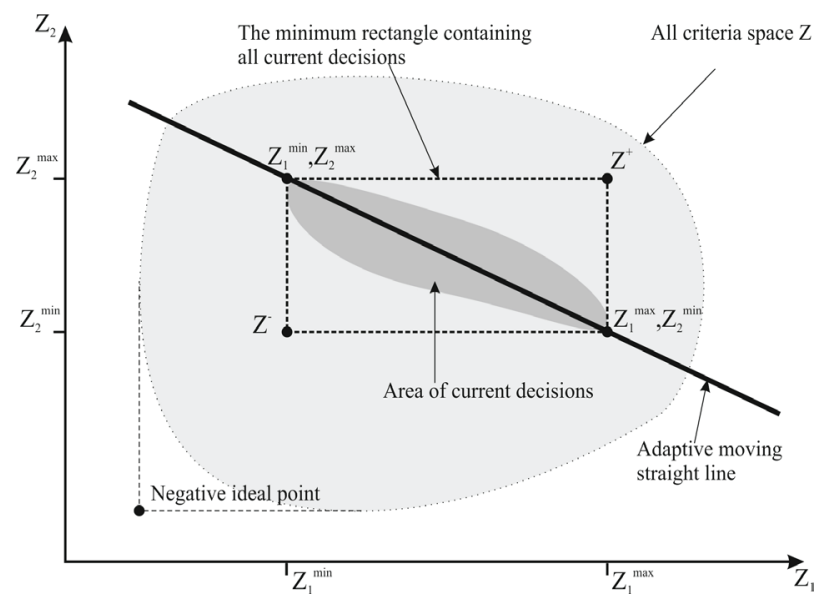

Fig.4. Adaptive weights and adaptive hyperplane in the space of criteria Z.

Since each the optimality criteria can have its own solution accuracy, which is achieved with different numbers of iterations $\left(n_{t}\right)$, for determining $n_{t}$ Experimental researches of all single-criterial problems were carried out and the number of iterations was determined, at which accuracy would be achieved corresponding to the technological conditions. Therefore, as a condition of stopping while solving a multicriteria problem, the maximum value from the total number of iterations is accepted.

Thus, the multicriteria optimization method, which was described above, allows one to search through the entire solution space for each ants population and choose those paths of motion that will be optimal in the sense of Pareto.

\section{Conclusions}

The variety of methods for constructing Pareto optimization points to the relevance of the problem were considered in this article. The development of this method in the direction of evolutionary and population optimization methods seems perspective.

The computational complexity of the particular optimality criteria is not the same in different parts the region of variable parameters and for tasks of schedule optimization the large dimension. Many modern Pareto optimization methods do not take this condition into account. In this regard, the development of adaptive and self-adaptive Pareto-optimization methods based on such promising optimization algorithms as the method of ant colonies is relevant.

The algorithm, which was proposed in this article for solving a multicriteria problem using the instrumental capabilities of the ant colony method makes it possible to construct an approximation not of the entire Pareto front, but of its part closest to the user-defined "preferred" point in the criteria space. This approach is realized by forming an integrated criterion, for example the sum of private criteria with some coefficients, where the "general" target function is constructed from separate target functions in the form of a weighted sum.

The properties inherent in ant algorithms contribute to the their effective application in solving multicriteria problems because ant algorithms are based on the use a multitude of potential solutions in the global search in various directions and do not impose any requirements on the form of target functions and constraints.

Thus, the proposed approach of the Pareto dominance concept, based on the ant algorithm, is of considerable interest.

\section{References}

1. E. Zitzler, K. Deb, L. Thiele, Evolutionary Computation Vol. 8(2), P. 173-195. (2000).

2. K. Deb, Multi-objective optimization using evolutionary algorithms (UK: Wiley. 2001)

3. S.Mostaghim, J. Teich. In: Swarm Intelligence Symposium, P. 26- 33 (2003)

4. D.Moor, D. Mukhlisullina, Science and education: electronic scientific and technical publication Vol. 4, P. 123-129 (2010)

5. V.Guliashki, H.Toshev, Ch.Korsemov, Problems of Engineering Cybernetics and Robotics Vol. 60, P. $42-54$ (2009)

6. V. Podinovsky, V. Nogin, Pareto-optimal solutions of multicriteria problems (RU: Moscow. 2007)

7. V. Nogin, Decision-making in a multicriteria environment: a quantitative approach. (RU: Moscow. 2005)

8. D.Ashlock, Evolutionary Computation for Modeling and Optimization (Springer. 2006)

9. I. Sobole, R. Statnikov, The choice of optimal parameters in problems with many criteria (RU: Moscow. 2006)

10. O. Chengar, Donbass-2020: Perspectives of development through the eyes of young scientists. P. 339-343 (2010)

11. J. Ryu, S. Kim, Proceedings of the 2009 Winter Simulation Conference (WSC), P. 623-633 (2009)

12. O. Chengar, Problems of Information Technology. Bulletin of Kherson National Technical University, Vol. 1(46). P. 212-217 (2013)

13. O. Chengar E. Savkova. Journal of East Ukrainian National University. 13 (167), P. 239-245 (2011).

14. Y. Skobtsov, A. Sekirin, S. Zemlyanskaya, O. Chengar, V. Skobtsov, S.Potryasaev, Advances in Intelligent Systems and Computing, vol.466, P.453-462 (2016)

15. Y. Skobtsov, O. Chengar, V. Skobtsov, A.Pavlov, Advances in Intelligent Systems and Computing, vol.573, P.456-465 (2017) 